\title{
Chronique de jurisprudence du droit des étrangers
} 2014

Journal of the Case Law of the Rights of Aliens 2014

\section{Guillaume Dujardin}

\section{(2) OpenEdition}

1 Journals

\section{Édition électronique}

URL : http://journals.openedition.org/crdf/1284

DOI : $10.4000 /$ crdf. 1284

ISSN : 2264-1246

\section{Éditeur}

Presses universitaires de Caen

\section{Édition imprimée}

Date de publication : 1 novembre 2015

Pagination : 163-172

ISBN : 978-2-84133-742-2

ISSN : 1634-8842

\section{Référence électronique}

Guillaume Dujardin, «Chronique de jurisprudence du droit des étrangers 2014 », Cahiers de la recherche sur les droits fondamentaux [En ligne], 13 | 2015, mis en ligne le 01 novembre 2016, consulté le 11

février 2020. URL : http://journals.openedition.org/crdf/1284; DOI : 10.4000/crdf.1284 


\title{
Chronique de jurisprudence du droit des étrangers 2014
}

\author{
Guillaume DUJARDIN \\ Doctorant en droit public à l'université de Caen Normandie \\ Centre de recherche sur les droits fondamentaux et les évolutions du droit (CRDFED, EA 2132)
}

\begin{abstract}
I. L'obtention du visa
A. L'obligation pour les Syriens de détenir un VTA: épilogue d'un feuilleton en manque de rebondissement

B. Le visa «mariage pour tous»

II. Le séjour en France: la non-invocabilité de la circulaire Valls

III. La défaite du droit d'être entendu préalablement à l'éloignement
\end{abstract}

"Contentieux de pauvres devenu contentieux de masse $»^{1}$, le droit des étrangers a encore occupé une place centrale dans les prétoires en 2014. Compte tenu de l'ampleur du contentieux et du nombre important des acteurs juridictionnels, il a été fait le choix pour cette nouvelle chronique de ne fonctionner que par "coups de projecteur» sur des sujets qui nous auront particulièrement marqué. Cette chronique ne sera donc pas frappée du sceau de l'exhaustivité... Pour cette "première», l'actualité sera divisée selon les trois temps de la «vie juridique» de l'étranger: son entrée sur le territoire - avec l'étape fatidique de l'obtention du visa - (I) ; son séjour - en l'occurrence les modalités de sa régularisation - (II); enfin, les garanties dont il dispose contre l'éloignement (III).

\section{L'obtention du visa}

L'année 2013 s'est clôturée avec l'épilogue de la «saga jurisprudentielle» du visa Schengen ${ }^{2}$. L'année 2014 sera marquée par deux affaires importantes, l'une concernait l'obligation faite aux Syriens de détenir un visa de transit aéroportuaire (VTA) pour faire escale en France (A), l'autre était relative à la délivrance d'un visa qui devait permettre à un couple homosexuel de se marier en France (B).

\section{A. L'obligation pour les Syriens de détenir un VTA: épilogue d'un feuilleton en manque de rebondissement}

Mauvais? Ce feuilleton sur l'obligation pour les Syriens de détenir un VTA n'a, en tout cas, pas connu de grand rebondissement... Quelques mots sont nécessaires pour saisir l'enjeu du problème. Le VTA, régi désormais par le Code communautaire des visas ${ }^{3}$, est une catégorie de visa de transit imposée aux ressortissants de certains États en vue de permettre leur passage par la zone internationale d'un aéroport. La liste des États de provenance soumis à ce visa est fixée à l'annexe IV du Code communautaire des

1. N. Fischer, «Contentieux de pauvres pour techniciens du droit», Plein droit, n 94, octobre 2012, en ligne: www.gisti.org/spip.php?article2949.

2. La question était en l'occurrence de savoir si l'étranger devait se présenter aux autorités compétentes lorsqu'il franchissait la frontière sous couvert d'un visa Schengen. Mettant un terme à l'incertitude jurisprudentielle, le Conseil d'État jugea que «la souscription de la déclaration prévue par l'article 22 de la convention d'application de l'accord de Schengen [...] est une condition de la régularité de l'entrée en France de l'étranger» (CE, avis, 18 décembre 2013, $\mathrm{n}^{\circ} 372832$ ).

3. Règlement (CE) no $810 / 2009$ du 13 juillet 2009 , art. 3 . 
visas; les États ayant en outre la possibilité, pour faire face à un afflux de migrants, de soumettre les ressortissants d'autres provenances à cette formalité (art. 3.2 du Code communautaire des visas). La question de soumettre certains ressortissants à ce visa est loin d'être neutre, humainement parlant. «Conçu[e] comme un instrument de lutte contre l'immigration illégale ${ }^{4}$, cette catégorie de visas vise entre autres à juguler les demandes d'asile massives 5 .

Afin d'étendre la liste fixée à l'annexe IV du règlement (CE) no 810/2009, a été adopté un arrêté interministériel le 10 mai 2010, soumettant les ressortissants de vingt-cinq nationalités à l'obtention d'un VTA. Puis, fin décembre 2012, le ministre des Affaires étrangères et le ministre de l'Intérieur firent connaître leur intention de soumettre, à compter du 15 janvier 2013, les ressortissants Syriens à l'obtention dudit visa. Ce n'est pourtant que par note du 22 janvier 2013 que le gouvernement français a notifié à la Commission européenne sa décision ${ }^{6}$. Il faudra alors attendre l'arrêté du 18 mars 2013 (publié le 21 au JORF) pour que l'article 2 de l'arrêté du 10 mai 2010 soit modifié à cette fin. C'est de la «décision» initiale (quelque peu «évanescente ${ }^{7}$ puisque révélée seulement par les sites Internet de certaines ambassades) dont il avait été demandé la suspension ${ }^{8}$ puis l'annulation, au motif entre autres ${ }^{9}$ de la violation de l'article 3.2 du règlement (CE) du 13 juillet 2009, et de l'atteinte portée au droit d'asile. Par un arrêt du 18 juin $2014^{10}$, le Conseil d'État clôt finalement notre «feuilleton» en donnant son «quitus» à la décision en question.

Ce sont les arguments tirés de l'atteinte au droit d'asile et de la méconnaissance des dispositions de l'article $3.2 \mathrm{du}$ Code communautaire des visas qui nous retiendront ici.

L'article 3.2 du règlement du 13 juillet 2009 dispose que

En cas d'urgence due à un afflux massif de migrants clandestins, chaque État membre peut exiger des ressortissants de pays tiers autres que ceux visés au paragraphe 1, qu'ils soient munis d'un visa de transit aéroportuaire.
Tout en opérant ici un contrôle de la qualification juridique des faits ${ }^{11}$, le Conseil d'État a retenu que compte tenu de «l'important exode de population » et du fait que nombre d'États membres (Belgique, République tchèque, Allemagne, Grèce, Espagne, Italie, Pays-Bas, Autriche) avaient soumis les Syriens à l'obligation de détenir le VTA, les conditions posées par l'article 3.2 étaient remplies. Si le deuxième argument utilisé peut se comprendre (en tant qu'indice), le premier laisse plus que dubitatif quant à son maniement. En dépit du nombre - en valeur brute - assez restreint de demandeurs d'asile syriens en France (de vingt en 2010, il était passé à cent quatre-vingts en $2012^{12}$ ) la haute juridiction semble n'avoir tenu compte que de la «tendance $»^{13}$ de l'évolution du nombre de demandes. Ce faisant, le durcissement du contrôle opéré par le Conseil d'État s'avère bien illusoire ${ }^{14}$. Surtout, l'utilisation de ce critère colle assez mal avec l'économie du dispositif prévu par l'article 3.2: il s'agit en effet de rechercher si l'afflux de migrants est de nature à entraîner des difficultés techniques dans la prise en charge de ces derniers.

S'agissant de l'atteinte portée au droit constitutionnel d'asile, force est de constater que la haute assemblée n'a pas apporté grande innovation. Dans la droite ligne de ses précédents en matière de VTA ${ }^{15}$, il a en effet été jugé que

[...] l'obligation de disposer d'un visa de transit aéroportuaire, qui ne peut être imposée par les États membres, en vertu du règlement du 13 juillet 2009, qu'en cas d'urgence due à un afflux massif de migrants clandestins et qui répond ainsi à des nécessités d'ordre public tenant à éviter, à l'occasion d'une escale ou d'un changement d'avion, le détournement du transit aux seules fins d'entrée en France, ne porte par elle-même aucune atteinte au droit d'asile ${ }^{16}$.

Le juge retient donc ici que ce n'est pas le fait d'élargir une liste de nationalités soumises à visa qui a pour effet de priver les intéressés de leur droit d'asile: libre à eux alors de solliciter la délivrance dudit visa en amont. Il reste évidemment toujours possible de s'interroger sur la

4. X. Domino, «La France peut exiger des Syriens un visa de transit aéroportuaire. Concl. sous CE, 18 juin 2014, Anafé et Gisti», L'actualité juridique. Droit administratif, $\mathrm{n}^{\circ} 30$, septembre 2014, p. 1714

5. Cet objectif est souligné par le Code communautaire des visas, cons. 5 .

6. Alors même que l'article 3.2 du règlement (CE) du 13 juillet 2009 indique que «Les États membres notifient à la Commission, avant qu'elles n'entrent en vigueur, ces décisions»...

7. C. Lantero, «De la validation du VTA par le Conseil d'État à la condamnation du refus de visa par le TA de Nantes», Lettre «Actualités DroitsLibertés» de La revue des droits de l'homme, 2 octobre 2014, en ligne: http://revdh.revues.org/886.

8. Recours rejeté par CE, ord., 15 février 2013, Anafé et Gisti, n 365709: Lettre "Actualités Droits-Libertés » de La revue des droits de l'homme, 3 mars 2013, comm. C. Lantero, en ligne: http://revdh.org/2013/o3/o3.

9. Dans le cadre de cette chronique, nous passerons sur les moyens de légalité externe développés par les associations requérantes (en dépit d'ailleurs de leur intérêt: voir C. Lantero, «De la validation du VTA...»).

10. CE, 18 juin 2014, Anafé et Gisti, no 366307, JurisData, n 2014-013536: X. Domino, «La France peut exiger des Syriens un visa de transit aéroportuaire...»; C. Lantero, «De la validation du VTA par le Conseil d'État...».

11. Il s'agit d'une innovation puisqu'avant cet arrêt, le Conseil se cantonnait au contrôle de l'erreur manifeste pour les décisions de soumettre les ressortissants de certains États à la détention d'un VTA. En ce sens, voir CE, 25 juillet 2008, Anafé et Gisti, n 313710.

12. En l'absence (étonnante!) de toute donnée chiffrée citée par le Conseil d'État ou son rapporteur public, on se rapporte à celles indiquées par C. Lantero dans «De la validation du VTA par le Conseil d’État...».

13. Comme l’y invitait son rapporteur public (voir ses conclusions précitées, X. Domino, «La France peut exiger des Syriens un visa de transit aéroportuaire...", p. 1717).

14. Le rapporteur public avouant lui-même que l'affichage par le juge de son intensité de contrôle n'allait "pas changer la substance de ce dernier» (ibid.).

15. CE, 25 juillet 2008, Anafé et Gisti; CE, ord., $1^{\text {er }}$ avril 2008, Anafé et Gisti, no 313711 (à propos d'une obligation de VTA faite aux ressortissants tchétchènes)

16. CE, 18 juin 2014, Anafé et Gisti, cons. 6. 
pertinence en pratique de ce raisonnement: une exigence de visa ne peut-elle pas aboutir à « intercepter» administrativement une demande d'asile, de même en quelque sorte qu'une interception en mer $^{17}$ ? Il est à reconnaitre que cette analogie ${ }^{18}$ s'avère assez lointaine dans la mesure où une obligation de remplir telle formalité administrative - en dépit des circonstances parfois troublées du pays - n'est pas, par elle-même, de nature à faire échec à un droit.

À l'évidence, Il en va différemment lorsque la décision contestée est un refus de visa opposé à une personne entendant solliciter l'asile. Dans un jugement du $16 \mathrm{sep}-$ tembre 2014, le tribunal administratif de Nantes juge que le refus de VTA opposé à la requérante porte, «dans les circonstances de l'espèce», une atteinte grave au droit constitutionnel d'asile et à son corollaire, le droit de solliciter le statut de réfugié ${ }^{19}$. Or, ce sont justement ces "circonstances particulières », à savoir que le visa a été refusé au motif que la demande d'asile de la requérante avait été rejetée en France (ce qui n'était pas le cas...), qui posent problème. Cette motivation implique en effet que c'est le consul qui a examiné la demande d'asile, ce qu'il n'a nullement compétence à faire, et c'est ce qui amène le tribunal administratif à annuler en l'espèce le refus de visa. Pourtant, cette assertion n'a - selon nous - pas lieu d'être car le refus de visa prive en lui-même l'intéressé de son droit de demander l'asile, d'autant qu'en l'espèce l'intéressée avait présenté sa demande de visa en indiquant son intention de faire valoir son droit à une protection internationale. Certes, ne censurer que les refus de visa sur le fondement du droit constitutionnel d'asile, et non la décision d'élargir la liste des pays soumis à VTA, pourrait apparaître "saugrenu». La décision individuelle n'estelle pas que le reflet, et la conséquence, de la décision "générale» initiale? Pas nécessairement. Il ne semble pas impossible de réduire les motifs de refus opposables par l'autorité consulaire aux seules menaces graves à l'ordre public et aux demandes manifestement frauduleuses de visa (hypothèse, il est vrai, très rare car il faudrait alors établir que l'intéressé entend entrer en France pour une raison autre que sa demande d'asile) ${ }^{20}$. De la sorte, il serait possible d'effectuer un contrôle strict sur la décision des autorités consulaires, sans nier frontalement la possibilité qu'a l'État de soumettre les ressortissants de certains États à l'obtention du VTA. Il est surtout à souligner que l'enga- gement dans cette voie ne demanderait pas un "grand effort » aux juridictions administratives. Elle aurait pour mérite de sauvegarder la cohérence de la jurisprudence sur le droit d'asile, tout en mettant la France à l'abri d'une condamnation par la Cour européenne des droits de l'homme (Cour EDH) ${ }^{21}$.

On ne peut d'ailleurs exclure que ce soit cette voie qu'ait en fait empruntée le tribunal de Nantes: la référence aux "circonstances de l'espèce» ne pourrait être qu'une erreur de plume, ou à tout le moins apparaître comme le motif le plus «sûr» de censure de la décision consulaire. Seule la jurisprudence à venir nous le dira...

\section{B. Le visa "mariage pour tous"}

L'ordonnance rendue par le Conseil d'État le 9 juillet $2014^{22}$ « corrobore le constat selon lequel le droit des étrangers fait partie des branches du droit dans lesquelles les libertés fondamentales pratiquent le concubinage avec les exigences sécuritaires ${ }^{23}$. En l'occurrence, un visa avait été refusé par les autorités consulaires françaises au Maroc à un ressortissant sénégalais voulant épouser un ressortissant français. Compte tenu de l'imminence de la célébration du mariage, le refus de visa a fait l'objet d'un référé-liberté (art. L. 521-2 du Code de justice administrative - CJA), recours rejeté par le tribunal administratif de Nantes, par une ordonnance du 24 juin 2014.

Deux difficultés se sont posées au Conseil d'État en l'espèce: la première concernait la condition de l'urgence, tandis que la seconde avait trait à la liberté de se marier ${ }^{24}$.

S'agissant de l'urgence au sens de l'article L. 521-2 du CJA, le juge administratif est assez réticent à la reconnaître pour les refus de visa, estimant très probablement que le référé-suspension (L. 521-1 CJA) offre une solution adaptée à ces espèces. Il est ainsi jugé

[...] qu'en principe, [...] le refus de délivrance d'un visa d'entrée sur le territoire français ne fait pas apparaître une situation qui justifie l'intervention à très bref délai d'une mesure de la nature de celles qui peuvent être ordonnées sur le fondement de l'article L. 521-2 du code de justice administrative; que la circonstance [...] qu'un long délai s'est écoulé entre la conclusion de son mariage et le refus de visa qui lui est opposé ne suffit pas à créer la situation d'urgence caractérisée ${ }^{25}$.

17. Cour EDH, GC, 23 février 2012, Hirsi Jamaa et autres c. Italie, $\mathrm{n}^{\circ} 27765 / 09$.

18. Elle a été faite notamment par C. Lantero dans son commentaire précité de cet arrêt.

19. TA Nantes, ord., 16 septembre 2014, $M^{m e}$ K. et autres, no 1407765.

20. Il est ici fait une analogie avec les deux dernières hypothèses dans lesquelles le préfet est en droit de refuser de délivrer une autorisation provisoire de séjour pour demande d'asile (L. 741-4 du Code de l'entrée et du séjour des étrangers et du droit d'asile - CESEDA, $3^{\circ}$ et $4^{\circ}$ ).

21. Le raisonnement tenu par la Cour EDH dans son arrêt Hirsi Jamaa et autres $c$. Italie pouvant probablement s'appliquer à l'hypothèse d'un refus de visa.

22. CE, ord., 9 juillet 2014, M. A., $\mathrm{n}^{\mathrm{o}} 382145$ : E. Aubin, «Le refus de délivrance d'un visa, le mariage pour tous et la liberté fondamentale de se marier. Note sous CE, ord., 9 juillet 2014, M. A.», L'actualité juridique. Droit administratif, nº 37, novembre 2014, p. 2141 sq.; I. Corpart, «Quand un refus de visa porte atteinte au droit au mariage, liberté fondamentale», Revue générale du droit, $\mathrm{n}^{\circ}$ 17532, en ligne: www.revuegeneraledudroit.eu/?p=17532.

23. E. Aubin, «Le refus de délivrance d'un visa...», p. 2143.

24. D'un point de vue procédural, cette affaire confirme en outre que la Commission de recours contre les refus de visa n'a pas à être saisie au préalable en cas d'exercice d'un référé-liberté. Voir notamment CE, ord., 18 décembre 2003, Ambdi Houmadi, nº 262654.

25. CE, ord., 8 octobre 2008, Alaattin A., nº 321248; voir également CE, ord., 18 février 2005, n 277579 
Il est d'ailleurs à souligner que cette condition d'urgence est appréciée avec une particulière fermeté. Le Conseil d'État a par exemple estimé que

[...] ni le fait que l'état de santé [du requérant] fasse obstacle à ce qu'il se déplace au Cameroun, ce qui rend impossible la célébration du mariage dans ce pays, ni la circonstance que la date librement choisie pour l'union projetée ait été fixée au 30 septembre 2005, ne permettent de caractériser une situation d'urgence particulière ${ }^{26}$.

Le Conseil d'État aurait-il donc fait preuve d'une bienveillance particulière en jugeant, dans notre affaire, que «compte tenu de la proximité de la date du mariage» la condition de l'urgence était en l'espèce remplie ${ }^{27}$ ? Pareille conclusion serait sans doute un peu rapide. L'espèce Nkoyock et Hazera était en effet très particulière, la requérante ayant déjà entrepris une demande de visa court séjour sans indiquer aux autorités consulaires l'imminence de la date de son mariage. À ceci s'était peut-être rajouté le fait que les deux futurs époux ne s'étaient, au préalable, jamais rencontrés... D’ailleurs, dans une espèce où le requérant venait de perdre sa fille (âgé de dix jours), le Conseil d'État a conclu qu'il y a une «urgence particulière» au sens des dispositions précitées car les circonstances «imposent qu'il puisse se rendre en France afin de rendre les derniers devoirs à sa fille et de pourvoir à l'organisation des obsèques ${ }^{28}$.

C'est donc de manière somme toute classique que le Conseil d'État a considéré la condition de l'urgence remplie en l'espèce. La condition de l'atteinte grave et manifestement illégale à une liberté fondamentale se posait, elle, sous un jour nouveau.

En soi, il ne faisait guère de doute que la liberté de se marier rentrait dans le champ d'application de l'article L. 521-2 du CJA. Jusqu'ici il est vrai, elle n'avait été consacrée comme telle que de façon implicite : le Conseil d'État se contentant de relever que la décision contestée ne portait pas atteinte à la liberté du requérant de se marier ${ }^{29}$. Mais, fondamentalement cette solution s'imposait. Reconnue explicitement à l'article 12 de la Convention européenne des droits de l'homme (Convention EDH) et considérée par le Conseil constitutionnel comme une composante des libertés individuelle ${ }^{30}$ et personnelle ${ }^{31}$, la liberté de se marier avait bien tous les traits d'une «liberté fondamentale». C'est donc en faisant preuve d'une audace bien mesurée que le Conseil d'État juge ici que «le refus de visa opposé par le Consul général de France [...] porte une atteinte grave à l'exercice par [le requérant] de la liberté de se marier, laquelle est une liberté fondamentale ${ }^{32}$.
L'intérêt «essentiel» de l'arrêt commenté a trait à l'extension du champ d'application de la liberté de se marier aux couples homosexuels. Nouvelle, cette question n'a pourtant pas obligé le Conseil d'État à franchir une marche trop haute... L'article 171-9 du Code civil prévoit en effet que

[...] lorsque les futurs époux de même sexe, dont l'un au moins a la nationalité française, ont leur domicile ou leur résidence dans un pays qui n'autorise pas le mariage entre deux personnes de même sexe et dans lequel les autorités diplomatiques et consulaires françaises ne peuvent procéder à sa célébration, le mariage est célébré publiquement par l'officier de l'état civil de la commune de naissance ou de dernière résidence de l'un des époux ou de la commune dans laquelle l'un de leurs parents a son domicile ou sa résidence établie.

C'est donc en interprétant conformément la liberté de se marier avec ces dispositions du Code civil - c'est d'ailleurs par elles que le juge a commencé son raisonnement - que le Conseil d'État franchit cette courte marche.

Finalement, la question la plus délicate portait sur la condition de «l'atteinte grave et manifestement illégale» à la liberté fondamentale. Le ministre de l'Intérieur avait fait valoir que la demande de visa poursuivait d'autres buts que la seule célébration du mariage, ainsi que l'attestaient, selon lui, la forte différence d'âge entre les deux futurs époux ( 35 ans), l'obligation de quitter le territoire français dont l'intéressé avait fait l'objet en 2007, et enfin le visa qui lui avait été refusé en 2013 par les autorités belges. Pour rejeter ces arguments, le juge du Palais-Royal a retenu d'une part que les deux futurs époux vivaient ensemble depuis plusieurs années au Maroc, et d'autre part que le requérant disposait d'un emploi stable dans ce pays. Et, le mariage ne pouvant être célébré ni par les autorités marocaines ni par le consul de France au Maroc, ne s'offrait d'autre possibilité à l'administration que de délivrer le visa sollicité afin de permettre au mariage de se tenir en France.

Si la solution retenue emporte la conviction, la haute juridiction laisse pourtant une question en suspens: quid d'une situation dans laquelle les futurs mariés auraient la volonté de se marier en France alors que rien ne fait obstacle à ce que leur union soit célébrée à l'étranger? Dans ce cas, il est probable qu'une décision de refus de visa ne soit pas considérée comme portant une atteinte grave à la liberté de se marier. Il est ainsi jugé qu'un refus de visa court séjour opposé à un ressortissant marocain, alors même que deux des sœurs de la future mariée étaient dans l'impossibilité de se rendre au Maroc, ne porte pas

26. CE, ord., 28 septembre 2005, Nkoyock et Hazera, n ${ }^{\circ} 285505$.

27. La circonstance que le mariage était prévue de longue date (les démarches en vue de se marier en France avaient été engagées dès l'été 2013; tandis que la demande de visa a été faite le 26 mai 2014) n'est sans doute pas pour rien dans la conclusion retenue.

28. CE, ord., 20 avril $2009, \mathrm{n}^{\circ} 327162$.

29. CE, ord., 13 janvier 2003, n' 253216; CE, ord., 7 août 2003, nº 259242; CE, ord., 23 août 2011, nº 351994 .

30. CC, déc. $n^{\circ} 93-325$ DC du 13 août 1993, cons. 3 .

31. CC, déc. $\mathrm{n}^{\circ} 2003-484$ DC du 20 novembre 2003, cons. 94

32. CE, ord., 9 juillet 2014, M. A., cons. 5 . 
une atteinte excessive au droit garanti par l'article 8 de la Convention $\mathrm{EDH}^{33}$. Nul doute que la solution retenue s'imposerait également pour la liberté de se marier.

Au final, ce que vient de consacrer le Conseil d'État s'approche plus d'un «droit au mariage» que d'une «liberté de se marier » : ce qui est garanti n'est en aucun cas le lieu du mariage, mais uniquement la possibilité in fine qu'il ait lieu. L'audace aura donc trouvé rapidement ses limites, sans que la Cour européenne des droits de l'homme puisse d'ailleurs reprocher grand-chose à cette tendance ${ }^{34}$.

\section{Le séjour en France: la non-invocabilité de la circulaire Valls}

La question de l'invocabilité contentieuse de la circulaire Valls ${ }^{35}$ aura été l'une de celles qui auront le plus divisé les juridictions administratives au cours de l'année 2014, de même qu'agité la blogosphère ${ }^{36}$. En gros, deux camps s'y sont opposés. Le premier visait à reconnaître que certaines des dispositions de la circulaire du 28 novembre 2012 constituent des "lignes directrices ${ }^{37}$, au sens de la jurisprudence Crédit foncier de France ${ }^{38}$, alors que le second le niait en bloc.

C'est cette seconde voie que décide d'emprunter le Conseil d'État. Dans son récent arrêt de section $M$. Cortes Ortiz $z^{39}$, la haute juridiction opine que dans le cas où l'administration peut légalement accorder une mesure de faveur, elle a la possibilité de déterminer des orientations générales, mais dont l'intéressé ne saurait se prévaloir dans le cadre d'un recours pour excès de pouvoir ${ }^{40}$. Il en découle
[...] que s'il peut, à l'appui d'un recours pour excès de pouvoir formé contre une décision préfectorale refusant de régulariser sa situation par la délivrance d'un titre de séjour, soutenir que la décision du préfet, compte tenu de l'ensemble des éléments de sa situation personnelle, serait entachée d'une erreur manifeste d'appréciation, il ne peut utilement se prévaloir des orientations générales que le ministre de l'intérieur a pu adresser aux préfets pour les éclairer dans la mise en œuvre de leur pouvoir de régularisation ${ }^{41}$.

Le Conseil d'État censure ici les positions audacieuses adoptées par les juges du fond dans cette affaire ${ }^{42}$.

Cet arrêt est intéressant à un double point de vue. Si d'un point de vue pratique, il met fin à une «longue» période d'incertitude jurisprudentielle ${ }^{43}$, c'est surtout de son intérêt théorique dont il convient ici de discuter. Revenant sur le célèbre «Lazare juridique » ${ }^{44}$ des directives "Crédit Foncier de France» (ci-après «directives $\mathrm{CFF}$ »), le présent arrêt se prononce sur la question du champ d'intervention des «lignes directrices». L'utilisation de cet instrument, promue par le Conseil d'État dans son étude annuelle précitée, s'il s'étend au-delà du domaine économique et fiscal ${ }^{45}$, peut-il servir de terre d'accueil aux « circulaires de régularisation» d'étrangers? La jurisprudence traditionnelle se montrait très réticente sur ce point ${ }^{46}$.

Jusqu'à cette circulaire du 28 novembre 2012 en effet, le Conseil d'État avait systématiquement rejeté pour inopérant le moyen tiré de la violation de dispositions d'une circulaire de «régularisation». Souvent, il le fit en se contentant de rappeler leur absence de valeur réglementaire ${ }^{47}$. Parfois,

33. CE, 21 mars $2007, \mathrm{n}^{\circ} 285609$.

34. Rappelons, s'il est besoin, que l'article 8 ne garantit pas un droit au séjour pour les étrangers: la cellule familiale pouvant en principe se constituer (ou se reconstituer) en France comme à l'étranger. Voir notamment: Cour EDH, 28 mai 1985, Abdulaziz, Cabales et Balkandali c. Royaume-Uni, $\mathrm{n}^{\circ}$ 92214/80, \$ 68. Et, C.-A. Chassin, «La vie familiale des étrangers », Cahiers de la recherche sur les droits fondamentaux, $\mathrm{n}^{\circ}$ 11, 2013, p. 77-85, spéc. p. $83-84$.

35. Circulaire du ministre de l'Intérieur du 28 novembre 2012, $\mathrm{n}^{\circ}$ NOR INTK1229185C.

36. Voir S. Slama, «L'invocabilité des lignes directrices dans les procédures de régularisation de sans-papiers. Note sous CAA Paris, 4 juin 2014», L'actualité juridique. Droit administratif, $\mathrm{n}^{\circ}$ 31, septembre 2014, p. 1773.

37. CE, Le droit souple, étude annuelle 2013 du Conseil d'État, Paris, La documentation française, 2013.

38. CE, sect., 11 décembre 1970, Crédit foncier de France c. Demoiselle Gaupillat et Dame Ader: Dalloz, 1971, p. 674, note D. Lochak; Revue du droit public, 1971, p. 1224, note M. Waline; Les grands arrêts de la jurisprudence administrative, $19^{\mathrm{e}}$ éd., Paris, Dalloz, 2013, p. 579.

39. CE, sect., 4 février 2015, Ministre de l'Intérieur c. M. Cortes Ortiz, no 383267 : L'actualité juridique. Droit administratif, n 4 , février 2015, p. 191, obs. D. Poupeau.

40. Ibid., cons. 4

41. Ibid., cons. 6 .

42. TA Paris, 18 décembre 2013, n 1306958 : L'actualité juridique. Droit administratif, n 8, mars 2014, p. 454, concl. A. Fort-Besnard. CAA Paris, 4 juin 2014, no 14PAo0358: L'actualité juridique. Droit administratif, 2014, nº 31, septembre 2014, p. 1773, note S. Slama.

43. Dans le sens de l'invocabilité de la circulaire, voir également CAA Bordeaux, 31 décembre 2014, n 14BXoo726; CAA Douai, 31 décembre 2014, $n^{\circ}$ 14DA01079; CAA Nancy, 18 décembre 2014, n 14NCoo395; CAA Marseille, 25 novembre 2014, n 13 MAo3584. Dans le sens de l'ininvocabilité de la circulaire, voir CAA Lyon, 2 octobre 2014, Lika, Dema et Haouaiji, no 14LYo1524, n 14 LYo1523, no 14LYo1620: L'actualité juridique. Droit administratif, $\mathrm{n}^{\circ} 37$, novembre 2014, p. 2112, note A. Samson-Dye; Revue française de droit administratif, n 6, novembre-décembre 2014, p. 1039, concl. L. Levy Ben Cheton; voir aussi CAA Nantes, 31 décembre 2014, n 14 NToogo1 (cette fois, en se fondant sur l'absence de valeur réglementaire de la circulaire); de même dans CAA Versailles, 18 décembre 2014, $\mathrm{n}^{\circ}$ 14VEo2030.

44. CE, 12 décembre 1997, Office national interprofessionnel des fruits, des légumes et de l'horticulture, n 147007: Droit administratif, février 1998, p. 7, concl. J.-H. Stahl.

45. Il est significatif de noter que la première utilisation de l'expression «ligne directrice» dans la jurisprudence du Conseil d'État s'est faite à l'occasion d'un litige extérieur à ces deux domaines (en l'occurrence, l'octroi de bourses pour les enfants scolarisés à l'étranger): CE, 19 septembre 2014 M. A., $\mathrm{n}^{\circ} 364385$, à paraître au Lebon: L'actualité juridique. Droit administratif, nº 32, septembre 2014, p. 1798, obs. J.-M. Pastor.

46. Pour une présentation exhaustive de cette jurisprudence, voir les conclusions d'A. Fort-Besnard précitées.

47. CE, 22 février 1999, Époux Useyin, n 197243 (à propos de la circulaire du 24 juin 1997 relative à la régularisation de certaines catégories d'étrangers en situation irrégulière); CE, 24 novembre 2010, Ministre de l'Intérieur c. Époux Korkmaz, nº 310885 (à propos de la circulaire du 13 juin 2006 relative à la régularisation de ressortissants étrangers ayant au moins un enfant scolarisé en France); CE, 31 juillet 1992, Préfet de Seine-et-Marne c. Kizil, nº 132778 (à propos de la circulaire du 23 juillet 1991 prévoyant la régularisation de certains étrangers déboutés dans leur demande d'asile). 
et de manière plus assurée, la haute juridiction affirmait que les étrangers en situation irrégulière ne bénéficient pas d'un droit subjectif à la régularisation de leur séjour, droit qu'une circulaire serait en outre impuissante à leur reconnaître ${ }^{48}$.

S'inscrivant donc clairement dans le cadre du second courant, la (riche) motivation de l'arrêt Cortes Ortiz semble opérer un amalgame fâcheux entre «mesure gracieuse » et "régularisation» d'un étranger ${ }^{49}$. En témoigne la rédaction de la fin du considérant 6 :

[...] il [l'intéressé] ne peut utilement se prévaloir des orientations générales que le ministre de l'intérieur a pu adresser aux préfets pour les éclairer dans la mise en œuvre de leur pouvoir de régularisation ${ }^{50}$.

$\mathrm{Si}$, de jurisprudence constante, l'autorité administrative n'est pas tenue (sauf disposition expresse contraire) de refuser à un étranger la délivrance d'un titre de séjour alors qu'il ne remplit pas les conditions légales, «la faculté pour le préfet de prendre [...] une mesure favorable à l'intéressé pour régulariser sa situation relève de son pouvoir d'appréciation $»^{51}$.

Pourtant, et ainsi que l'illustre très bien l'article L. 313$11,7^{\circ}$ du CESEDA, le pouvoir de « régulariser» les étrangers ne s'identifie pas nécessairement avec les hypothèses de délivrance gracieuse d'un titre de séjour. Prise dans son sens juridique commun, l'action de régulariser recouvre «l'action de purger [...] une situation du vice formel qui l'entache en réparant celui-ci par une initiative positive qui consiste précisément, en général, en l'accomplissement de la formalité adéquate ${ }^{52}$. Pour ne pas dénaturer les mots, il aurait sans doute été plus pertinent de distinguer suivant que la circulaire renvoie à l'article L. 313-11, $7^{\circ}$ (pts 2.1.1 à 2.1.3 de la circulaire du 28 novembre 2012) ou à l'article L. 313-14 du CESEDA (pts 2.1.4 et 2.2 de la même circulaire). La problématique juridique est radicalement différente dans ces deux hypothèses. La première sera de savoir si des «lignes directrices » peuvent apparaître là où l'autorité décisionnaire se trouve dans une situation de compétence liée, alors que la seconde amènera à se questionner sur la possibilité d'adopter de telles «directives» alors que l'intéressé ne dispose d'aucun droit subjectif à l'octroi de l'avantage souhaités3.

Dans la première hypothèse, la reconnaissance de «lignes directrices» nous semble délicate sans être complètement impossible. Ainsi que l'indiquait L. Levy Ben Cheton, l'interprétation de la loi (L.313-11, $\left.7^{\circ}\right)$ à la lumière de la jurisprudence

[...] pourrait éventuellement revêtir une portée impérative [...], mais à la condition alors, pour l'auteur de la circulaire, de veiller [...] à ne pas dénaturer le contenu de la jurisprudence, dont il ne saurait évidemment durcir le sens ${ }^{54}$.

Dans ce cas, il faudra alors que les termes de la circulaire soient suffisamment précis, suffisamment directifs, pour encadrer l'administration dans sa prise de décision. C'est probablement dans cet esprit que la cour administrative d'appel de Paris ${ }^{55}$, sur conclusions pourtant contraires de son rapporteur public, a décelé des «lignes directrices» dans le point 2.1.1 ${ }^{56}$. On pourrait objecter à cette analyse qu'en dehors des cas où l'article 8 de la Convention EDH (tel qu'interprété par la jurisprudence) implique la délivrance d'un titre de séjour, octroyer tout de même ce dernier reviendrait à faire usage d'un pouvoir de régularisation. Néanmoins, cette dernière hypothèse peut tout aussi bien s'analyser comme une "dérogation", la jurisprudence admettant ici parfaitement l'usage de directives $\mathrm{CFF}^{57}$.

La reconnaissance de pareilles «lignes directrices » semble en revanche exclue, dans son principe, s'agissant du pouvoir gracieux du préfet. C'est d'ailleurs ce que vient rappeler le Conseil d'État dans le considérant 4 de son arrêt. Juger autrement reviendrait en effet à ce que la circulaire octroie à l'intéressé un droit subjectif au séjour, alors que le législateur en a décidé autrement. Le «droit au séjour» serait alors octroyé par une autorité incompétente. Une analogie peut ici être faite avec l'arrêt Gisti du 23 octobre 2009. Saisie notamment de la légalité

48. CE, 26 octobre 1990, Ntrika, no 111859 (à propos de la circulaire du 5 août 1987 par laquelle le ministre de l'Intérieur invitait, sous certaines conditions, les préfets à régulariser les personnes dont la demande d'asile a été rejetée); CE, 29 décembre 1993, Banzuzi, n 141318 (à propos de la circulaire de 1991 précitée). Les termes de cet arrêt méritent d’être rappelés: «cette circulaire n’avait pas pour objet et n’aurait pu légalement avoir pour effet de poser des règles de droit nouvelles, ni de limiter le pouvoir qui appartenait à l'administration, en l'absence de toute règle de droit le lui interdisant, de régulariser la situation des étrangers".

49. Cet «amalgame» s'explique sans doute par des raisons «historiques». Antérieurement à la création de l’article L. 313-11, $7^{\circ}$ du CESEDA, le pouvoir de régulariser un étranger s'arrêtait aux seules hypothèses de mesures gracieuses. Le Conseil d'État, dans un important avis de 1996, estimait ainsi: «il ne peut exister un "droit à la régularisation", expression contradictoire en elle-même. La régularisation, par définition, est accordée dans l'hypothèse où le demandeur d'un titre de séjour ne bénéficie pas d'un droit, sinon il suffirait qu'il le fasse valoir» (CE, avis, 22 août 1996, $\mathrm{n}^{\circ}$ 359.622: Les grands avis du Conseil d'État, $3^{\mathrm{e}}$ éd., Paris, Dalloz, 2008, p. 345).

50. Dans l'affaire ici commentée, l'intéressé se prévalait d'ailleurs des dispositions de l'article L. 313-11, $7^{\circ}$ du CESEDA.

51. CE, sect., 4 février 2015, Ministre de l'Intérieur c. M. Cortes Ortiz, cons. 5.

52. Vocabulaire juridique, G. Cornu (dir.), $3^{\mathrm{e}}$ éd., Paris, PUF, 1992, p. 697.

53. Pour un raisonnement en ce sens, voir L. Levy Ben Cheton dans ses conclusions précitées.

54. Ibid., p. 1040-1041.

55. CAA Paris, 4 juin 2014, précité.

56. Le rapporteur public ne contestait d'ailleurs pas ici le principe même que la circulaire puisse contenir de telles «lignes directrices », mais notait simplement que les orientations contenues au point 2.1.1 lui paraissaient trop floues, «molles», «creuses»... (voir la note de S. Slama, «L’invocabilité des lignes directrices...», p. 1777).

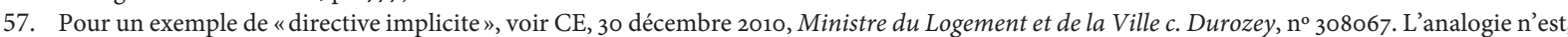
il est vrai, qu'imparfaite dans la mesure où l'article L. 313-11, $7^{\circ}$ ne prévoit pas expressément qu'il peut lui être dérogé. Elle ne perd néanmoins pas de sa pertinence dans la mesure où c'est le juge qui, ici, aura décerné à l'administration ce pouvoir. 
de la circulaire du 7 janvier 2008, la haute juridiction estime que le ministre

[...] ne pouvait, sans méconnaître la loi, restreindre les conditions de délivrance de cette carte de séjour temporaire en subordonnant la recevabilité des demandes de délivrance de cette carte à la présentation, par l'étranger, d'une promesse d'embauche dans l'un des métiers prévus par cette liste; [...] par suite [...], le requérant est fondé à demander l'annulation de cette circulaire comme entachée d'incompétence ${ }^{58}$.

De façon plus générale, cet arrêt tend bien à accréditer la thèse qu'une " telle formalisation, par voie de circulaire, des modalités d'instruction des demandes d'admission exceptionnelle ne saurait légalement avoir pour objet, ou pour effet, de retirer sa nature gracieuse à la compétence préfectorale ${ }^{59}$.

Exception faite de cette «réserve», le principe même qu'il ne saurait y avoir de «lignes directrices» dans les hypothèses de pouvoir gracieux ne nous semble pas contestable. Reste alors à savoir si l'article L. 313-14 du CESEDA laisse à l'administration un pouvoir discrétionnaire, ou s'il n'est que l'officialisation du pouvoir gracieux du préfet en matière de régularisation ${ }^{60}$. C'est la réponse à cette question - aussi fondamentale que délicate - qui dictera l'issue du litige.

Aux termes de l'article L. 313-14 en effet, une carte de séjour temporaire

[...] peut être délivrée, sauf si sa présence constitue une menace pour l'ordre public, à l'étranger ne vivant pas en état de polygamie dont l'admission au séjour répond à des considérations humanitaires ou se justifie au regard des motifs exceptionnels qu'il fait valoir, sans que soit opposable la condition prévue à l'article L. 311-7 (nous soulignons).

Les expressions «considérations humanitaires» ou, dans une moindre mesure, «motifs exceptionnels » pourraient laisser penser, malgré leur extrême plasticité, à des «conditions» dictant l'attribution d'un droit. Analysé ainsi, l'article L. 313-14 n'est plus simplement la formalisation du pouvoir gracieux dont jouit naturellement l'autorité administrative en matière de régularisation, mais bien un titre de séjour distinct, entouré de certaines conditions.
Le considérant retenu par la cour administrative d'appel de Paris dans une affaire $M^{m e} L{ }^{6}{ }^{61}$ semble aller en ce sens:

[...] par les énonciations précitées [pt 2.2.2 de la circulaire], le ministre de l'intérieur a, sans limiter le pouvoir d'appréciation des préfets dans l'application des dispositions de l'article L. 313-14 [...] ni le pouvoir discrétionnaire de régulariser la situation d'un étranger qui leur appartient, indépendamment de ces dispositions, [...] défini des orientations générales applicables à la délivrance d'une carte de séjour temporaire ${ }^{62}$.

Pour des raisons, certes différentes, il apparaît donc que le raisonnement du Conseil d'État dans cette affaire Cortes Ortiz est contestable. Ni l'article L. 313-11, $7^{\circ}$, ni l'article L. 313-14 n'étant à proprement parler les émanations du pouvoir gracieux du préfet, les raisons pour dénier en bloc la qualification de «lignes directrices " à la circulaire du 24 novembre 2012 demeurent assez fragiles. S'écarter ainsi des propositions qu'il a formulées dans son rapport annuel pour $2013^{63}$ semble alors malheureux, d'autant qu'en pratique ces circulaires de «régularisation» sont perçues par l'administration elle-même comme de véritables «normes», dont il ne lui appartiendrait de s'écarter que de façon exceptionnelle.

\section{La défaite du droit d'être entendu préalablement à l'éloignement}

Le droit d'être entendu préalablement à une décision d'éloignement aura marqué la fin de l'année 2014. Consacré tant par les principes généraux du droit de l'Union que par l'article 41.2 sous a), de la Charte des droits fondamentaux (CDF), et invoqué inlassablement devant les juridictions du fond (notamment à l'encontre d'obligations de quitter le territoire français - OQTF), la Cour de justice de l'Union européenne (CJUE) vient de préciser son étendue s'agissant de ces dernières ${ }^{64}$. Les faits de l'espèce méritent ici d'être rappelés. Arrivée en France en septembre 2009 afin d'y effectuer une demande d'asile, et déboutée de cette

58. CE, 23 octobre 2009, Gisti, $\mathrm{n}^{\circ} 314397$, mentionné dans les tables du recueil Lebon, p. 791.

59. Conclusions de L. Levy Ben Cheton précitées, p. 1044

60. La différence entre les deux situations est, il est vrai, très ténue. Reprenant largement à l'analyse du rapporteur public dans l'arrêt de la cour administrative d'appel de Lyon. On pourrait dire que le pouvoir discrétionnaire recouvre des situations dans lesquelles un intéressé peut prétendre au bénéfice d'un droit, dont néanmoins les conditions d'obtention sont très imprécises de sorte que l'autorité jouit d'un large pouvoir d'appréciation. À l'inverse, une compétence gracieuse s'analyse en une simple mesure de bienveillance pour laquelle l'intéressé ne saurait revendiquer aucun droit acquis. Sur cette question, voir L. Levy Ben Cheton, conclusions précitées, p. 1049-1051; B. Pacteau, "Discrétionnarité», in Dictionnaire de la culture juridique, $4^{\mathrm{e} e ́ d ., ~ D . ~ A l l a n d, ~ S . ~ R i a l s ~(d i r .), ~ P a r i s, ~ P U F, ~ 2012, ~ p . ~} 374$.

61. CAA Paris, 20 juin 2014, Préfet de police c. $M^{m e} L ., \mathrm{n}^{\circ} 14$ PAoooo1: L'actualité juridique. Droit administratif, $\mathrm{n}^{\circ}$ 27, juillet 2014, p. 1541, obs. L. Boissy.

62. Ibid., cons. 12 (nous soulignons). Les termes utilisés par la cour administrative d'appel nous semblent tout à fait significatifs puisqu'elle distingue clairement le pouvoir de régulariser un étranger en l'absence de texte - sa compétence étant ici «discrétionnaire» - et celui de lui accorder un titre de séjour sur le fondement de l'article L. 313-14 - dans ce cas, l'administration ne jouissant plus que d'un "pouvoir d'appréciation» (ce qui impliquerait un «degré de discrétionnarité» largement moindre).

63. Il y indique en effet que tant pour l'octroi de la nationalité française que pour l'admission exceptionnelle au séjour «le préfet dispose d'un pouvoir d'appréciation qu'il est utile d'orienter par voie de lignes directrices [...]. De fait, les circulaires applicables dans ce domaine ont à bien des égards les caractéristiques de lignes directrices" (CE, Le droit souple, p. 144-145).

64. CJUE, 5 ch., 5 novembre 2014, Sophie Mukarubega c. préfet de police et préfet de la Seine-Saint-Denis, aff. C-166/13: L'actualité juridique. Droit administratif, n 6, février 2015, p. 334, chron. E. Broussy, H. Cassagnabère et C. Gänser; Lettre «Actualités Droits-Libertés» de La revue des droits de l'homme, 18 novembre 2014, comm. M.-L. Basilien-Gainche et T. Racho, en ligne: http://revdh.revues.org/957. 
demande par une décision de la Cour nationale du droit d'asile (CNDA) du 30 août 2012, la requérante s'est vue en conséquence notifier une OQTF le 26 octobre 2012 (assortie d'un délai de départ volontaire - DDV - d'un mois). Puis, s'étant maintenue irrégulièrement sur le territoire français, tout en cherchant à partir pour le Canada sous couvert d'un passeport d'emprunt - ce qui lui valut d'être mise en garde à vue -, $\mathrm{M}^{\mathrm{me}}$ Mukarubega a fait l'objet d'une seconde OQTF (le 5 mars 2013). Elle a en outre été placée, par un arrêté du même jour, dans un centre de rétention administrative. Le tribunal administratif de Melun a, par un jugement du 8 mars $2013^{65}$, annulé pour défaut de base légale l'arrêté de placement en rétention, et posé à la Cour de justice deux questions préjudicielles. Par la première, le juge demande en substance si le droit d'être entendu - tel que protégé par les principes généraux du droit de l'Union et repris à l'article 41 de la CDF - implique que l'intéressée soit mise en mesure de présenter ses observations préalablement à l'édiction de la décision de retour. La seconde consistait quant à elle, en cas de réponse positive de la Cour à la première question, à savoir si le caractère suspensif du recours juridictionnel permettait de déroger au droit d'être entendu.

L'intérêt de l'arrêt Mukarubega est double: d'une part, il permet de clore toute spéculation sur l'applicabilité de ce principe aux OQTF, et d'autre part, il précise l'étendue du droit d'être entendu préalablement à une mesure d'éloignement.

La réponse à la première question n'avait, il est vrai, rien d'évident. Proclamé à l'article 41.2 sous a), le droit d'être entendu préalablement à l'édiction d'une «mesure individuelle qui l'affecterait défavorablement» ne semble être opposable qu'aux institutions, organes et organismes de l'Union (art. 41.1). C'est ce qui explique que de nombreux juges du fond ont estimé - en retenant des expressions quelque peu différentes - que cette disposition ne permettait pas à l'étranger d'opposer à l'administration un droit à être entendu préalablement à toute mesure d'éloignement ${ }^{66}$. Mettant ainsi fin à toute incertitude, la Cour tranche: «il résulte clairement du libellé de l'article 41 de la Charte que celui-ci s'adresse non pas aux États membres, mais uniquement aux institutions, aux organes et aux organismes de l'Union [...] ${ }^{67}$. L'avocat général dans ses conclusions avait pourtant émis un avis contraire dans la mesure où l'article 51 de la Charte indique que les droits fondamentaux qu'elle vient proclamer s'appliquent dès lors que le droit de l'Union est mis en œuvre. De la sorte, «il ne [lui] paraîtrait pas cohérent ni conforme à la jurisprudence de la Cour que le libellé de l'article 41 de la Charte puisse ainsi introduire une exception à la règle prescrite par l'article 51 de celle-ci ${ }^{68}$. Ce débat s'avère pourtant largement théorique: le droit d'être entendu fait en effet «partie intégrante du respect des droits de la défense, principe général du droit de l'Union ${ }^{69}$.

La question cruciale sera donc de savoir si les étrangers pourront bénéficier d'un droit d'être entendu avant que l'autorité préfectorale n'adopte son arrêté portant obligation de quitter le territoire. Et c'est justement là où le bât blesse dans notre arrêt. Pourtant, en ellemême, la base du raisonnement de la Cour pourrait se comprendre. La prémisse tient ici dans l'article 6.1 de la directive $2008 / 115 / \mathrm{CE}^{70}$ : ce dernier, tel qu'interprété par une jurisprudence établie de la Cour, instaure un lien quasi indissociable entre le caractère irrégulier du séjour et la prise d'une mesure d'éloignement ${ }^{71}$. De cette prémisse la Cour fait découler la conséquence que, dès lors que l'intéressé a eu l'occasion d'être entendu dans le cadre de sa «demande de séjour ou, le cas échéant, sur l'irrégularité de son séjour ${ }^{72}$, il n'a pas à l'être spécifiquement dans le cadre de la procédure d'éloignement ${ }^{73}$. Ce point de vue rencontre un écho favorable dans la jurisprudence administrative française. Certaines cours administratives d'appel ont ainsi jugé que le droit d'être entendu

[...] n'implique pas systématiquement l'obligation, pour l'administration, d'organiser, de sa propre initiative, un entretien avec l'intéressé, ni même d'inviter ce dernier à produire ses observations, mais suppose seulement que, informé de ce qu'une décision lui faisant grief est susceptible d'être prise à son encontre, il soit en mesure de présenter spontanément des observations écrites ou de solliciter un entretien pour faire valoir ses observations orales ${ }^{74}$.

De façon parfaitement similaire, le Conseil d'État estime d'ailleurs que

[...] dans le cas prévu au $3^{\circ}$ du I de l'article L. 511-1 du code de l'entrée et du séjour des étrangers et du droit d'asile, où la décision faisant obligation de quitter le territoire français est prise concomitamment au refus de délivrance d'un

65. TA Melun, 8 mars 2013, Sophie Mukarubega c. préfet de police et préfet de la Seine-Saint-Denis, n 1301686/12: Lettre "Actualités Droits-Libertés» de La revue des droits de l'homme, 19 mars 2013, comm. S. Slama, en ligne: http://revdh.org/2013/03/19.

66. Voir les références jurisprudentielles citées par M.-L. Basilien-Gainche et T. Racho dans leur commentaire précité.

67. CJUE, $5^{\mathrm{e}}$ ch., 5 novembre 2014, Sophie Mukarubega c. préfet de police et préfet de la Seine-Saint-Denis, pt 44.

68. Ibid., pt 56 des conclusions. Cette position rencontrait d'ailleurs un écho assez favorable dans la doctrine. En ce sens: D.-U. Galetta, «Le champ d'application de l'article 41 de la Charte des droits fondamentaux de l'Union européenne sur le droit à une bonne administration. À propos des arrêts Cicala et M. ", Revue trimestrielle de droit européen, $n^{\circ}$ 1, janvier-mars 2013, p. 77 ; J. Ziller, « Droit à une bonne administration ", Jurisclasseur Libertés, mis à jour en 2007, fasc. 1040, $\$ 70$.

69. Ibid., pt 45 de l'arrêt.

70. Directive 2008/115/CE du 16 décembre 2008.

71. CJUE, ${ }^{\text {re }}$ ch., 28 avril 2011, El Dridi, aff. C-61/11, pt 35; CJUE, GC, 6 décembre 2011, Achughbabian c. préfet du Val-de-Marne, aff. C-329/11, pt 31 ( Une fois constatée l'irrégularité du séjour, lesdites autorités doivent, en vertu de l'article 6, paragraphe 1, de ladite directive et sans préjudice des exceptions prévues par cette dernière, adopter une décision de retour »; nous soulignons).

72. CJUE, $5^{\mathrm{e}}$ ch., 5 novembre 2014, Sophie Mukarubega c. préfet de police et préfet de la Seine-Saint-Denis, pt 62.

73. Ibid., pt 60 .

74. CAA Lyon, 14 mars 2013, M. Halifa, $n^{\circ} 12$ LYo2704, cons. 8 ; raisonnement similaire dans CAA Paris, 31 juillet 2013 , $M^{m e}$ Tometey, $\mathrm{n}^{\circ} 12 \mathrm{PAo} 2 \mathrm{O} 4 \mathrm{O}$ 
titre de séjour, l'obligation de quitter le territoire français découle nécessairement du refus de titre de séjour; que le droit d'être entendu n'implique alors pas que l'administration ait l'obligation de mettre l'intéressé à même de présenter ses observations de façon spécifique sur la décision l'obligeant à quitter le territoire français, dès lors qu'il a pu être entendu avant que n'intervienne la décision refusant de lui délivrer un titre de séjour ${ }^{75}$.

Le raisonnement ainsi opéré peine à convaincre: le lien indissociable créé entre mesure d'éloignement et décision sur le séjour est très relatif. Si ce lien peut à la limite s'opérer entre refus d'accorder une protection internationale et décision d'éloignement (comme dans l'affaire Mukarubega), il est nettement plus délicat à faire quand il s'agit d'une demande de séjour pour raison familiale ou professionnelle (par exemple). Dans ces derniers cas, les questions que poseront le séjour et l'éloignement pourront être distinctes, et impliqueront des observations bien spécifiques (par exemple au regard de l'article 3 de la Convention EDH pour la décision d'éloignement). Ce faisant, la Cour semble perdre de vue le but même du droit d'être entendu, tel que dégagé dans son arrêt Sopropé de 2008 : ce droit

[...] a pour but que l'autorité compétente soit mise à même de tenir utilement compte de l'ensemble des éléments pertinents. Afin d'assurer une protection effective de la personne ou de l'entreprise concernée, elle a notamment pour objet que ces derniers puissent corriger une erreur ou faire valoir tels éléments relatifs à leur situation personnelle qui militent dans le sens que la décision soit prise, ne soit pas prise ou qu'elle ait tel ou tel contenu ${ }^{76}$.

Alors qu'elle reprend pourtant cette formule de principe au point 47 de son arrêt, la Cour paraît ici n'en tirer aucune conséquence. Faisant une application radicale de l'adage «nul n'est censé ignorer la loi », elle part du postulat que l'étranger savait qu'en cas de refus de séjour, il serait visé par une OQTF, et qu'il était par ce fait même en mesure de présenter des observations quant à un éventuel éloignement au moment de l'examen de son droit de séjour... L'utilisation de cette fiction conduira immanquablement à priver en pratique l'administration d'éléments pertinents pour l'étude du dossier, faisant du même coup apparaître le droit d'être entendu comme bien "théorique et illusoire».

Néanmoins, dans un arrêt rendu le 11 décembre $2014^{77}$, la Cour semble revenir à davantage de fermeté. Confrontée il est vrai à des faits un peu différents ${ }^{78}$, la Cour exige que l'intéressé soit invité à s'exprimer sur les modalités de son retour ${ }^{79}$; et plus généralement sur les motifs qui pourraient inciter l'administration à renoncer à la mesure d'éloignement ${ }^{80}$.

Est-ce à dire que l'arrêt Mukarubega ne valide en rien la position du Conseil d'État dans son arrêt Halifa ${ }^{81}$ ? Ce n'est pas exclu... Au point 60 de son arrêt du 5 novembre, la Cour de justice prend tout de même le soin de préciser que l'autorité administrative doit mettre l'intéressé en mesure de présenter ses observations sur les motifs pouvant conduire l'administration à ne pas prononcer de mesure d'éloignement. Les exigences exactes de la Cour en la matière paraissent cependant bien floues, et la formule utilisée au point 60 n'invite pas à un grand optimisme.

En effet, la cour exige non pas que l'autorité administrative ait invité l'étranger à présenter ses observations, mais bien que ce dernier «a[it] eu la possibilité» de le faire. À prendre cette formule au pied de la lettre, le juge de Luxembourg semble simplement exiger que les «portes» de l'administration ne soient pas fermées à l'intéressé...

L'application des principes dégagés au cas d'espèce peut également étonner...

Ainsi, pour établir le respect du droit d'être entendu lors de la première décision d'éloignement, la Cour de justice retient que l'intéressée a été entendue tant par l'OFPRA que par la CNDA ${ }^{82}$. Or, «ces auditions n'ont porté exclusivement que sur les motifs d'éligibilité à une protection internationale, et en aucun cas sur le droit au séjour ou les conséquences d'une éventuelle OQTF ${ }^{83}$. Bien qu'en pratique l'appréciation sur le droit au séjour dépende exclusivement de l'appréciation portée par les autorités de l'asile sur la protection internationale, ces deux décisions sont juridiquement bien distinctes, et prises par des autorités différentes. Et ce d'autant que l'autorité préfectorale n'est jamais tenue - hors circonstances d'ordre public évidemment - de refuser de délivrer le titre de séjour ${ }^{84}$.

«Pire» encore, et c'est assurément là que l'arrêt désarçonnera le plus, la Cour se fonde sur l'audition en garde à vue afin d'établir la conformité de la seconde décision avec le droit d'être entendu. Pareille assertion ne pourra que désarmer l'observateur, en même temps que le laisser pessimiste quant à la «substance » même du droit d'être entendu. Les questions auxquelles doit répondre la police n'ont rien de commun avec celles que devront se poser les autorités préfectorales préalablement à la prise de la mesure d'éloignement. Plus encore, affirmer que dans le cadre de cette garde à vue, $\mathrm{M}^{\mathrm{me}}$ Mukarubega «a notamment été entendue sur son droit de séjour en

75. CE, 4 juin 2014, M. Halifa, $\mathrm{n}^{\circ} 370515$, cons. 7 : L'actualité juridique. Droit administratif, $\mathrm{n}^{\circ}$ 26, juillet 2014, p. 1501, concl. X. Domino; La semaine juridique, édition administrations et collectivités territoriales, $\mathrm{n}^{\circ} 51,22$ décembre 2014, p. 31, note G. Marti.

76. CJCE, $2^{\mathrm{e}}$ ch., 18 décembre 2008, Sopropé, aff. C-349/o7, pt 49; CJUE, $5^{\mathrm{e}}$ ch., 3 juillet 2014, Kamino International Logistics BV, aff. C-129/13, pt 38 .

77. CJUE, $5^{\mathrm{e}}$ ch., 11 décembre 2014, Khaled Boudjlida c. préfet des Pyrénées-Atlantiques, aff. C-249/13.

78. L'intéressé n'ayant ici pas fait de demande de titre de séjour.

79. CJUE, $5^{\mathrm{e}}$ ch., 11 décembre 2014, Khaled Boudjlida c. préfet des Pyrénées-Atlantiques, pt 51.

80. Ibid., pt 49.

81. CE, 4 juin 2014, M. Halifa.

82. CJUE, $5^{\mathrm{e}}$ ch., 5 novembre 2014, Sophie Mukarubega c. préfet de police et préfet de la Seine-Saint-Denis, pt 69.

83. Commentaire précité de M.-L. Basilien-Gainche et T. Racho.

84. Voir nos observations sur l'invocabilité de la circulaire Valls. 
France» ${ }^{85}$, en plus d'avoir "été invitée [...] à ajouter toutes les observations qu'elle considérait comme étant pertinentes $\gg{ }^{86}$ ne peut que surprendre. Et ce, pour la simple et bonne raison que les services de police sont radicalement incompétents pour se positionner quant au droit au séjour de l'intéressé, et encore plus sur l'opportunité juridique de son futur éloignement. On ne voit donc pas en quoi cette circonstance lui aura permis de présenter son point de vue de manière $"$ utile» ${ }^{87}$.

Il est donc peu de dire que le raisonnement de la Cour de justice ne convainc guère, puisqu'il semble ramener le droit d'être entendu à une simple possibilité théorique pour l'étranger de s'exprimer au cours de la procédure d'examen du titre de séjour. Et ce, d'autant plus que les exigences pratiques de la Cour de justice semblent ici très minces.

Plus fondamentalement, cette position a pour effet de priver le droit des étrangers d'encore un instrument pour juguler le contentieux ${ }^{88}$. Plutôt que d'appréhender le droit d'être entendu pour ce qu'il devrait / pourrait être - un instrument permettant de régler le litige en amont en offrant notamment la possibilité à l'administration de corriger une erreur matérielle -, la Cour semble le percevoir comme un simple moyen dilatoire pour l'étranger. Le sort contentieux du droit d'être entendu rejoint sur ce point celui réservé à la Commission des titres de séjour ${ }^{89}$. Ce sont donc bien les exigences de la «lutte contre l'immigration illégale» qui l'ont emporté sur le droit d'être entendu ${ }^{90}$.

85. CJUE, $5^{\mathrm{e}}$ ch., 5 novembre 2014, Sophie Mukarubega c. préfet de police et préfet de la Seine-Saint-Denis, pt 74.

86. Ibid., pt 75

87. Pour reprendre la formule de principe utilisée par la Cour ( «L droit d'être entendu garantit à toute personne la possibilité de faire connaître, de manière utile et effective, son point de vue au cours de la procédure administrative et avant l'adoption de toute décision susceptible d'affecter de manière défavorable ses intérêts"), ibid., pt 46; voir également CJUE, $1^{\text {re }}$ ch., 22 novembre 2012, M. M., aff. C-277/11, pt 87.

88. Voir sur ce point le commentaire précité de S. Slama sur le jugement du TA de Melun.

89. Alors que sa saisine est obligatoire lorsque le préfet envisage de refuser la délivrance d'un titre de séjour, en principe accordé de plein droit (L. 313-12 du CESEDA), la jurisprudence est venue peu à peu vider cet article de son effet utile. En le conditionnant au fait que l'étranger remplisse toutes les conditions pour l'obtention dudit titre de séjour (CE, 13 décembre 2013, nº 361575), le Conseil d'État est venu avaliser la pratique des cours administratives d'appel qui déduit de l'absence d'illégalité interne de la décision que l'article L. 313-12 n'a pas été méconnu (parmi beaucoup d'autres : CAA Bordeaux, 21 novembre 2012, $\mathrm{n}^{\circ}$ 12BXoo710; CAA Nancy, 5 avril 2012, $\mathrm{n}^{\circ}$ 11NCo1636; CAA Marseille, 6 décembre 2011, $\mathrm{n}^{\circ}$ 09MAo2507).

90. CJUE, $5^{\mathrm{e}}$ ch., 5 novembre 2014, Sophie Mukarubega c. préfet de police et préfet de la Seine-Saint-Denis, pt 71 de l'arrêt. 